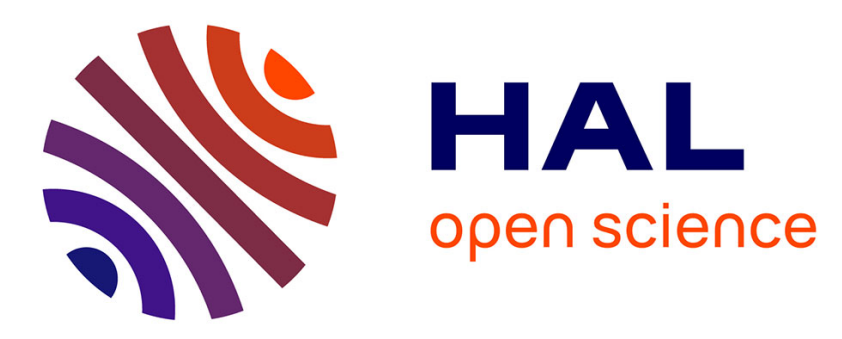

\title{
Efficient modelling of nonlinear propagation in multimode graded-index fibers
}

\author{
Matteo Conforti, C. Mas Mas Arabí, A. Bendahmane, Arnaud Mussot, \\ Alexandre Kudlinski
}

\section{> To cite this version:}

Matteo Conforti, C. Mas Mas Arabí, A. Bendahmane, Arnaud Mussot, Alexandre Kudlinski. Efficient modelling of nonlinear propagation in multimode graded-index fibers. Nonlinear Photonics, Jul 2018, Zurich, France. NpTh3C.2, 10.1364/NP.2018.NpTh3C.2 . hal-02393035

\section{HAL Id: hal-02393035 \\ https://hal.science/hal-02393035}

Submitted on 4 Dec 2019

HAL is a multi-disciplinary open access archive for the deposit and dissemination of scientific research documents, whether they are published or not. The documents may come from teaching and research institutions in France or abroad, or from public or private research centers.
L'archive ouverte pluridisciplinaire HAL, est destinée au dépôt et à la diffusion de documents scientifiques de niveau recherche, publiés ou non, émanant des établissements d'enseignement et de recherche français ou étrangers, des laboratoires publics ou privés. 


\title{
Efficient modelling of nonlinear propagation in multimode graded-index fibers
}

\author{
M. Conforti, C. Mas Arabí, A. Bendahmane, A. Mussot, and A. Kudlinski \\ Univ. Lille, CNRS, UMR 8523-PhLAM-Physique des Lasers Atomes et Molécules, F-59000 Lille, France \\ matteo.conforti@univ-lille1.fr
}

\begin{abstract}
We develop an effective 1+1D model describing nonlinear propagation in multimode graded-index fibers. The model is able to quantitatively reproduce recently observed phenomena like geometric parametric instability and broadband dispersive wave emission.
\end{abstract}

OCIS codes: (060.4370) Nonlinear optics, fibers; (060.5530) Pulse propagation and temporal solitons; (190.4420)

Nonlinear optics, transverse effects in.

Nonlinear pulse propagation in multimode fibers (MMFs) is focusing a tremendous research interest [1]. The experimental observations of multimode solitons generating ultrabroadband dispersive waves [2], geometric parametric instability (GPI) [3], and beam self-cleaning [4], are striking examples of the incredibly rich and complex scenario offered by nonlinear propagation in GRIN fibers. The two models exploited for the mathematical description of propagation in MMFs, nalemy the 3+1D Generalized Nonlinear Schrödinger Equation (GNLSE) [3], and the multi-mode GNLSE (MM-GNLSE) [5], are computationally very expensive. We describe here an efficient and accurate model for the description of nonlinear pulse propagation in parabolic GRIN fibers. It consist in a 1+1D GNLSE with a periodic nonlinear coefficient, which can be solved in an extremely efficient way by standard split-step methods and requires very modest computational resources. Our model accurately reproduces different phenomena peculiar to parabolic GRIN fibers, like geometric parametric instability and broadband dispersive wave emission [7]. The starting point of our investigations is the following 3+1D GNLSE [3]

$$
i \partial_{z} E+\frac{1}{2 \beta_{0}} \nabla_{T}^{2} E+d\left(i \partial_{t}\right) E-\frac{\beta_{0} \Delta}{r_{c}^{2}} r^{2} E+\frac{\omega_{0} n_{2}}{c} f_{N L}(E)=0
$$

where $r^{2}=x^{2}+y^{2}, \nabla_{T}^{2}=\partial_{x}^{2}+\partial_{y}^{2}$ is the transverse Laplacian, $E$ is the field envelope expressed in $\sqrt{\mathrm{W}} / \mathrm{m}$, $\beta_{0}=\omega_{0} n_{0} / c, n_{0}=n_{c o}$ and $r_{c}$ are the core radius and refractive index, $\Delta$ is the relative refractive index difference, $d\left(i \partial_{t}\right)=\sum_{n>2}\left(i \partial_{t}\right)^{n} \beta_{n} / n$ ! is the dispersion operator at the carrier frequency $\omega_{0}$. The function $f_{N L}(E)=$ $\left(1+i \tau_{s} \partial_{t}\right)\left[\left(1-f_{r}\right)|E|^{2} E+f_{r} E \int h_{r}\left(t^{\prime}\right)\left|E\left(t-t^{\prime}\right)\right|^{2} d t^{\prime}\right]$ describes the Kerr and Raman nonlinear responses, and $\tau_{s} \approx 1 / \omega_{0}$ is the self-steepening time. The propagation of a continuous wave beam in a parabolic GRIN fiber experiences selfimaging, due to the equal spacing of the propagation constant of the modes [1]. If the injected field is a Gaussian beam, the field remains approximately Gaussian. The amplitude of the solution takes the following form [6]:

$$
\left.\left|E_{s}(x, y, z)\right|=A_{s}\left|F_{s}(x, y, z)\right|=A_{s} \frac{a_{0}}{a(z)} \exp \left[-\frac{1}{2} \frac{r^{2}}{a^{2}(z)}\right], \quad a^{2}(z)=a_{0}^{2}\left[\cos ^{2}(\sqrt{g} z)+C \sin ^{2}(\sqrt{g} z)\right)\right]
$$

where $a_{0}$ is the beam spot size in $z=0, g=2 \Delta / r_{c}^{2}, A_{s}^{2}=2 p n_{0} /\left(n_{2} \beta_{0}^{2} a_{0}^{2}\right), C=(1-p) /\left(\beta_{0}^{2} a_{0}^{4} g\right), p$ being a dimensionless number measuring the distance from beam collapse. We write the solution of Eq. (1) as $E(x, y, z, t)=A(z, t) \cdot F_{s}(x, y, z)$, where we have approximated the stationary self-imaging field with its linear shape $\left[p \approx 0, C \approx 1 /\left(\beta_{0}^{2} a_{0}^{4} g\right)\right.$ in Eq. (2)], and accounted for all the temporal and nonlinear effects in the envelope $A(z, t)$. By inserting this Ansatz into Eq. (1), after multiplication by $F_{s}^{*}$, and integration over the transverse plane $x, y$, we get:

$$
i \partial_{z} \psi+d(i \partial t) \psi+\gamma(z) f_{N L}(\psi)=0, \quad \gamma(z)=\frac{\omega_{0} n_{2}}{c A_{e f f}(z)}=\frac{\omega_{0} n_{2}}{2 \pi c a^{2}(z)}
$$

where $\psi(z, t)=A(z, t) \sqrt{\mathscr{S}}$ is the envelope normalized to the area $\mathscr{S}=\iint\left|F_{S}(x, y, z)\right|^{2} d x d y=\pi a_{0}^{2}$, so that $|\psi|^{2}$ represents the optical power expressed in Watts, and $a^{2}(z)$ is given by Eq. (2). The effective area is obtained through the standard definition $A_{e f f}=\left(\iint\left|F_{s}(x, y, z)\right|^{2} d x d y\right)^{2} / \iint\left|F_{s}(x, y, z)\right|^{4} d x d y$. Equation (3) constitutes a 1+1D GNLSE where the spatial effects are summarized by the periodic nonlinear coefficient $\gamma(z)$. Physically, the self-imaging pattern 
generates a $z$-varying effective area $A_{e f f}(z)$, due to the periodic beam focusing, which thus couples the spatial evolution to the temporal envelope $\psi(z, t)$. Equation (3) perfectly reproduces complex spatiotemporal phenomena like GPI $[3,6]$ and ultrabroadband dispersive wave emission [2], and agrees quantitatively with 3+1D GNLSE [7].

In order to test the accuracy of our model on the field, we performed experiments in a 2 meters long commercial parabolic GRIN fiber (Draka 50/125). We pumped the fiber by a microchip laser, delivering 425 ps pulses at $1064 \mathrm{~nm}$ with the repetition rate of $1 \mathrm{kHz}$. The Gaussian pump beam was focused at the input face of the fiber with a FWHMI diameter of 12 microns and a peak power of $30 \mathrm{~kW}$. Figure 1 shows the measured output spectrum (blue curve), which is perfectly reproduced by numerical solution of Eq. (3) (dashed red curve). For the numerical simulation we used the full dispersion curve of $0.1 \%$ Germanium doped silica, and the parameters reported in the caption of Fig. 1. The perfect agreement between simulation and experiment allow us to unambiguously identify the different spectral peaks. The two main peaks at $\pm 130 \mathrm{THz}$ are the first GPI bands (band number 1, green lines). The first GPI band turns out to be phase-matched also at $180 \mathrm{THz}$ through higher order dispersion (band number 2, cyan line). The line at $235 \mathrm{THz}$ (band number 3, magenta line) is due to the cascaded GPI [8] generated by the intense spectral component at $130 \mathrm{THz}$. The peak at $260 \mathrm{THz}$ derives from the four-wave mixing between the pump and the first GPI band (band number 4, grey line). The peak at $80 \mathrm{THz}$ is not generated by nonlinear effects, and is a residue of the diode pumping the laser.

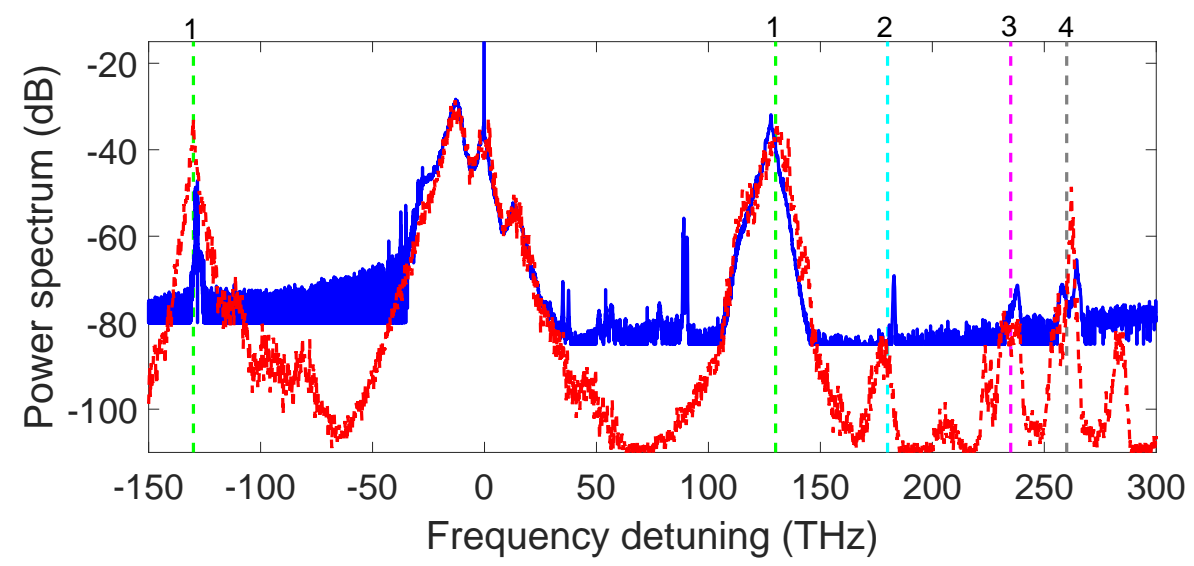

Fig. 1. Measured (blue curve) and simulated (red dashed curve) spectrum after the propagation in 2 meters of a commercial GRIN fiber. Simulation parameters: $P=30 \mathrm{~kW}$, pulse duration $40 \mathrm{ps}$ (supergaussian), $r_{c}=26 \mu \mathrm{m}, \Delta=0.0091, n_{2}=3.2 \cdot 10^{-20} \mathrm{~m}^{2} / \mathrm{W}, a_{0}=10 \mu \mathrm{m}$.

To conclude, we have derived a new model for the description of nonlinear propagation in parabolic GRIN fibers, which quantitatively reproduces experiments on GPI. In general, it is able to describe all the scenarios where a stable self-imaging pattern is generated inside the fiber. Given the drastic reduction of computational time with respect to the full 3+1D equation, we expect that our model will become a workhorse for the description of nonlinear dynamics in MMFs.

\section{References}

1. A. Mafi, J. Light. Technol. 30, 2803 (2012).

2. L. G. Wright, S. Wabnitz, D. N. Christodoulides, and F. W. Wise, Phys. Rev. Lett. 115, 223902 (2015).

3. K. Krupa, A. Tonello, A. Barthélémy, V. Couderc, B. M. Shalaby, A. Bendahmane, G. Millot, and S. Wabnitz, Phys. Rev. Lett. 116, 183901 (2016).

4. K. Krupa, A. Tonello, B. M. Shalaby, M. Fabert, A. Barthélémy, G. Millot, S. Wabnitz, and V. Couderc, Nat. Photonics 11, 237241 (2017).

5. F. Poletti and P. Horak, J. Opt. Soc. Am. B 25, 1645 (2008).

6. S. Longhi, Opt. Lett. 28, 2363 (2003).

7. M. Conforti, C. Mas Arabí, A. Mussot, and A. Kudlinski, Opt. Lett. 42, 4004 (2017).

8. R. Dupiol, A. Bendahmane, K. Krupa, A. Tonello, M. Fabert, B. Kibler, T. Sylvestre, A. Barthélémy, V. Couderc, S. Wabnitz, and G. Millot,Opt. Lett. 42, 1293 (2017). 